\title{
Flexible weighting of target features based on distractor context
}

\author{
Jeongmi Lee ${ }^{1} \cdot$ Joy J. Geng ${ }^{2,3}$ \\ Published online: 18 November 2019 \\ (C) The Psychonomic Society, Inc. 2019
}

\begin{abstract}
Models of attention posit that attentional priority is established by summing the saliency and relevancy signals from featureselective maps. The dimension-weighting account further hypothesizes that information from each feature-selective map is weighted based on expectations of how informative each dimension will be. In the current studies, we investigated the question of whether attentional biases to the features of a conjunction target (color and orientation) differ when one dimension is expected to be more diagnostic of the target. In a series of color-orientation conjunction search tasks, observers saw an exact cue for the upcoming target, while the probability of distractors sharing a target feature in each dimension was manipulated. In one context, distractors were more likely to share the target color, and in another, distractors were more likely to share the target orientation. The results indicated that despite an overall bias toward color, attentional priority to each target feature was flexibly adjusted according to distractor context: RT and accuracy performance was better when the diagnostic feature was expected than unexpected. This occurred both when the distractor context was learned implicitly and explicitly. These results suggest that featurebased enhancement can occur selectively for the dimension expected to be most informative in distinguishing the target from distractors.
\end{abstract}

Keywords Attention: Selective, Attention · Theoretical and Computational Models · Visual search

The ability to selectively attend to a subset of objects is necessary in natural environments where more information is present than can be processed. Cognitive models of attention have long theorized that attentional priority is established by summing the saliency and relevancy signals from featureselective maps (Cave \& Wolfe, 1990; Treisman \& Gelade, 1980). For example, if searching for an apple, attentional priority to "red" within the color map and "round" within a shape map would be summed, and attention would be directed to the location of the "red round" object that best matches the desired apple. These theories posit that attentional priority is determined in two steps-first by what is salient and relevant

Jeongmi Lee

jeongmi@kaist.ac.kr

1 Graduate School of Culture Technology, Korea Advanced Institute of Science and Technology, 291 Daehak-ro, Yuseong-gu,

Daejeon 34141, South Korea

2 Center for Mind and Brain, University of California, Davis, 267 Cousteau Pl, Davis, CA 95618, USA

3 Department of Psychology, University of California, Davis, 135 Young Hall, One Shields Avenue, Davis, CA 95616, USA within a feature dimension, and then based on the combined signals across feature maps (Treisman \& Gelade, 1980; Treisman \& Sato, 1990; Wolfe, 1994). While these models have provided mechanistic predictions that have received substantial cognitive and neurophysiological evidence (Hamker, 2004; Maunsell \& Treue, 2006; Treue \& Martinez Trujillo, 1999; Wolfe, 1994; Wolfe \& Horowitz, 2004), studies have not fully tested how selection mechanisms within this framework are biased by the diagnostic value of different feature dimensions within an object. Yet it is important to consider the informational value of an object's feature dimensions because real world objects tend to be complex collections of many features, some of which are more diagnostic of the target than others. For example, the "redness" and "roundness" are not equally diagnostic of apples situated within a basket full of other round fruits such as oranges, peaches, and grapefruits. In such a distractor context, the color dimension is more informative than the shape dimension.

Feature integration theory (FIT) was the first cognitive model that provided a full description of the mechanisms of attentive visual search (Treisman \& Gelade, 1980). FIT posited the existence of preattentive maps for specific feature dimensions (e.g., color, orientation) that fed into a master spatial map on which spatial attention operated to bind features into a 
single object representation. Importantly, targets that could not be automatically selected based on a unique feature (e.g., a red target among green) must be selected by attentive serial inspection of each object at the level of the master spatial map. Targets defined by a conjunction of features (e.g., a red $T$ amongst green $T \mathrm{~s}$ and red $L \mathrm{~s}$ ) are a classic example of the type of target that requires serial search. FIT therefore theorized that targets are either selected rapidly based on the uniqueness of a single feature (i.e., pop-out) or serially when the targets are highly similar to distractors or when targets are defined by a combination of two (or more) features. Many findings and subsequent attentional models have built on the core ideas of FIT, which have provided more nuanced descriptions of how information accrual occurs for target and distractor features (Wolfe, 2007; Wolfe, Cave, \& Franzel, 1989).

One particularly interesting subsequent theory is the "dimension-weighting" account of visual attention, which considers the diagnostic value of target feature dimensions as a function of surrounding distractors. The dimension-weighting account hypothesizes that information from each target feature is weighted based on expectations of how informative each dimension will be (Müller, Heller, \& Ziegler, 1995; Töllner, Müller, \& Zehetleitner, 2012; Töllner, Zehetleitner, Gramann, \& Müller, 2010). For example, Müller et al. (1995) asked subjects to search for a target defined by three features (e.g., small horizontal gray bar) among distractors. One target feature was always unique from distractors (e.g., all distractors were vertical and the target horizontal), resulting in pop-out. Importantly, the dimension of the pop-out was reliable (e.g., always in orientation) in some blocks or varied across dimensions (i.e., orientation, color, or size) in other blocks. The distractors in all conditions were identical and always shared two features with each target. The results showed consistent reaction time (RT) benefits when the target feature that popped-out was always from a single dimension compared with when it varied across dimensions. Even though the target always popped out from distractors along one dimension, there was a cost when the dimension of the pop-out switched or was unpredictable. These studies suggest that attention is weighted toward the more diagnostic feature dimension such that all features within the selected dimension are treated as equivalent, and distinct from information in other dimensions (Krummenacher \& Müller, 2012; Liesefeld \& Müller, 2019; Müller et al., 1995; Müller, Reimann, \& Krummenacher, 2003; Weidner \& Müller, 2009).

Despite providing clear evidence for dimensional weighting, the theory stops short of describing how dimensional and feature-specific information are combined to enhance processing. On the surface, dimensional weighting might seem to contradict the theories of feature-based enhancement. However, because feature dimensions are more abstract categories of information than features, there are several ways in which the ability to bias attention toward dimensions may occur in addition to enhancement of target features. One possibility is that the two operate on different distractor contexts. The dimensional-weighting account hypothesizes that dimensional biases arise when the target can be detected from distractors based on a "difference" in their signals within a single feature dimension map without needing to know where or what exactly is that difference. As such, it may be that dimensional weighting occurs only when it is unnecessary to know the exact feature of the target, given the distractor context, whereas feature-based weighting occurs when exact target features are necessary to identify the target. An alternative possibility is that dimensional weighting might combine with feature-based mechanisms to make visual search more efficient by selectively up-weighting attentional enhancement of the more informative feature from a multifeature target (Wolfe, 2007).

The current set of studies address whether information encoding and attentional selection of a conjunction target (defined by color and orientation) is biased by expectations about the more informative feature dimension, even when the exact target features are known in advance. Evidence for this would support the notion that we use dimensional information to selectively enhance feature-based attentional processing to optimize visual search.

In all experiments, each trial began with a "cue" stimulus of an upcoming conjunction target (defined by one of three colors and one of three orientations), with the exact features of the target changing on every trial. Different from the classic conjunction search paradigm, distractors that shared each of the target features were not present simultaneously within a single trial, but instead were present across different trials, and the probability of distractors sharing the target feature from one of two dimensions was manipulated across conditions. In other words, unlike other studies of dimensional weighting, participants were asked to find a new conjunction target that was indicated in advance by a cue on every trial, but similar to those studies, the distractor context set expectations for the most diagnostic information. Thus, even though the specific target features changed from trial-to-trial, one feature dimension was more likely to be diagnostic of the target. If dimensional information is used to select targets in response to expectations of distractor context, then we would expect lowfrequency targets that violate expectations about the diagnostic feature to interfere with performance.

Knowing the dimension of greater diagnostic value in a conjunction target helps prioritize feature selection to the most predictive value. We hypothesize that expectations based on the probability of distractors sharing a target feature will drive attention to weight the diagnostic feature more heavily than the less informative one, resulting in better performance when expectations are valid than invalid. However, one complication of testing this question involves possible asymmetries in 
perceptual or attentional sensitivity to information from different dimensions. For example, it is known that color information tends to have precedence over other feature dimensions (Biderman, Biderman, Zivony, \& Lamy, 2017; Geng, DiQuattro, \& Helm, 2017; Lee, Leonard, Luck, \& Geng, 2018; Theeuwes, 1991, 1992; White, Lunau, \& Carrasco, 2014). Thus, the first experiment was conducted to measure differences in the a priori bottom-up strength of each dimension to capture attention (pop-out search) in the context of our paradigm. Subsequent experiments then used a more complex design that required serial search in order to measure relative changes in attentional weighting of each feature dimension as a function of the distractor context.

\section{Experiment 1}

In Experiment 1, we conducted a conceptual replication of previous work on dimensional weighting, but with the explicit goal of documenting the differences in the a priori bias toward color versus orientation information in our paradigm. We examined whether feature values of a conjunction target are differentially weighted by the likelihood that the feature dimension is diagnostic of the target. The target was always a conjunction stimulus indicated by a cue before a visual search array. The critical manipulation was in the distractor set: In the color-weighted distractor context, the target was more likely to be different from distractors in color, whereas in the orientation-weighted distractor context, the target was more likely to differ from distractors in orientation. We hypothesized that, consistent with the use of dimensional weighting to narrow down feature-based gain modulation, observers will learn to bias attention toward features from dimensions that are more diagnostic of the target. However, we also expect overall differences in the strength of bias to color versus orientation. Attentional biases to one target feature was measured by comparing performance on trials consistent with expectations based on the distractor context (i.e., valid trials) against trials that violate expectations (i.e., invalid trials).

\section{Method}

Participants Twenty-three participants ranging in age from 18 to 24 years (10 males, mean age: 20.22 years) engaged in a 1-hour session for course credit. The number of participants was determined a priori by a power analysis ( $\mathrm{G} *$ Power, Version 3.1.9.2; http://www.gpower. hhu.de/), with .80 power to find a medium-size effect in a repeated-measures within-factors ANOVA test. All had normal or corrected-to-normal vision and gave informed consent. All of the experimental procedures were approved by the Institutional Review Board of the University of California, Davis.
Apparatus and stimuli Stimuli were presented on a Dell 2408WFP monitor (refresh rate $=60 \mathrm{~Hz}$ ) using Psychopy software (Version 1.83.4; http://www.psychopy.org/). The participants viewed the monitor from a distance of $70 \mathrm{~cm}$ in a dimly lit room. The monitor had a black background ( 0.31 $\left.\mathrm{cd} / \mathrm{m}^{2}, x=.31, y=.42\right)$ and contained a gray fixation cross (11. $7 \mathrm{~cd} / \mathrm{m}^{2}, x=.30, y=.33$ ) that was visible at all times unless occluded by the cue. Participants were instructed to maintain fixation throughout the experiment. For color stimuli, we chose three colors (orange, green, blue) among 360 equally spaced colors that only varied in hue in CIELAB space $\left(\mathrm{L}^{*}=\right.$ 70 , center: $\mathrm{a}^{*}=0, \mathrm{~b}^{*}=0$, radius of 38 ; from Bae, Olkkonen, Allred, \& Flombaum, 2015). Each target color was 90 degrees of visual angle from the neighboring target color.

Design and procedure Each participant performed a visual search task (see Fig. 1), in which the target was an elliptical sinusoidal grating $\left(1.7^{\circ} \times 2.9^{\circ}\right.$ in diameter, 3 cycle/degree in spatial frequency, Gaussian mask) defined by the combination of a specific color and orientation. For each trial, the color of the target was randomly chosen from orange $\left(18.5 \mathrm{~cd} / \mathrm{m}^{2}, x=\right.$ $.42, y=.40)$, green $\left(18.8 \mathrm{~cd} / \mathrm{m}^{2}, x=.30, y=.44\right)$, and blue $\left(18.9 \mathrm{~cd} / \mathrm{m}^{2}, x=.23, y=.28\right)$, and the orientation of the target was randomly chosen from $50^{\circ}, 90^{\circ}$, and $130^{\circ}$.

Each trial started with a cue display of $1,000 \mathrm{~ms}$, in which one of the nine possible combinations of color and orientation (e.g., green $50^{\circ}$ ) was shown on the center of the monitor, indicating the target to search for in that trial. Following $500 \mathrm{~ms}$ of fixation display, the search display was presented for $500 \mathrm{~ms}$, followed by another fixation display until response. The search display consisted of four items, with each item located at one of four positions (top left, top right, bottom left, bottom right) that was $2.56^{\circ}$ away from the fixation cross. Participants responded whether the target was present or absent in the search display as quickly and accurately as possible, by pressing the $n$ or $m$ key on the keyboard, respectively.

There were two trial types based on the feature match between the target and distractor items (see Fig. 1). On color trials, distractors were the same orientation as the target, but were different in color, such that participants had to discriminate between colors to find the target. On these trials, the color of the distractors was randomly chosen from the pool of possible target colors (orange, green, and blue), excluding the actual target color on that trial. On orientation trials, distractors were the same color as the target, but were different in orientation, such that participants had to discriminate between orientations to find the target. The orientation of the distractor items was randomly chosen from the pool of possible target orientations $\left(50^{\circ}, 90^{\circ}\right.$, and $\left.130^{\circ}\right)$, excluding the target orientation on that trial.

The three distractor items in a search display were all identical, enabling pop-out search on the diagnostic dimension. Half of all trials contained the target in the search display 
a

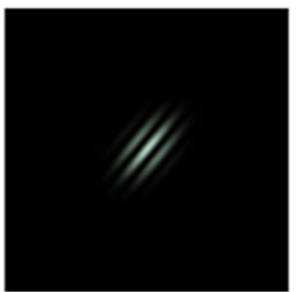

Cue

$1,000 \mathrm{~ms}$
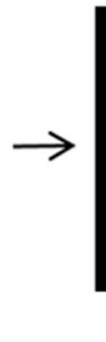

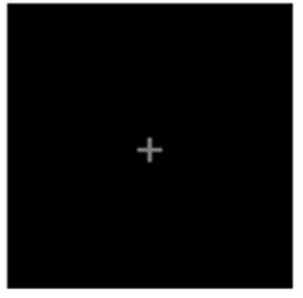

Fixation

$500 \mathrm{~ms}$

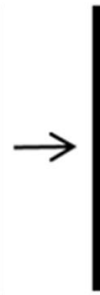

Search display
$500 \mathrm{~ms}$

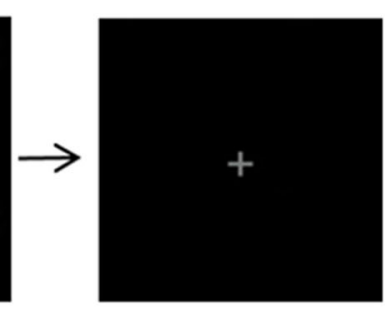

Response

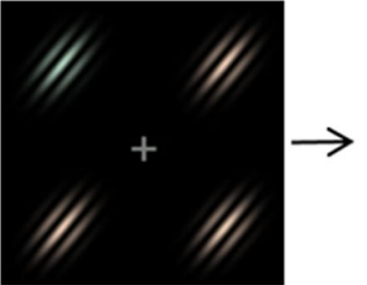

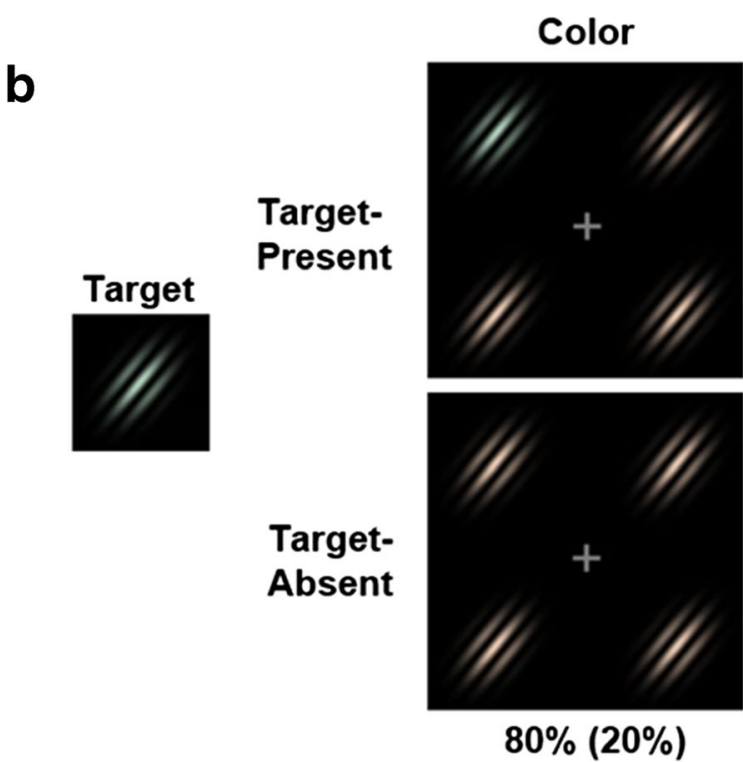

Fig. 1 a Sequence of events and time course of a trial in the visual search task. On each trial, an exact cue of the upcoming conjunction target defined by a combination of a specific color and orientation was presented, and participants pressed one of two keys to indicate whether the target was present or absent in the search display. In this specific example, the target was a green $50^{\circ}$ tilted grating. b Example search displays for different trial types in Experiment 1. The distractor items were all identical, enabling pop-out search. In color trials, distractors had the same orientation but different color with the target. In

(target present) and the other half did not (target absent). The location of the target and distractor items in the search display was randomly determined.

Importantly, the percentage of color trials and orientation trials was manipulated across conditions of distractor context: the color-weighted distractor context consisted of $80 \%$ color trials and $20 \%$ orientation trials, whereas the orientation-weighted distractor context consisted of $20 \%$ color trials and $80 \%$ orientation trials. Participants were not explicitly told about the probability of each trial type. Eight consecutive color-weighted distractor context blocks and eight consecutive orientation-weighted distractor context blocks were presented ( 40 trials/block, 16 blocks), with the order of distractor contexts counterbalanced across participants. Participants were encouraged to take a break after each block.

\section{Orientation}
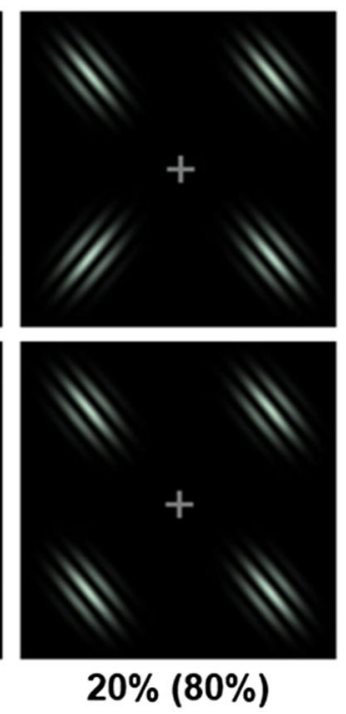

orientation trials, distractors had the same color, but different orientation with the target. The half of each trial type contained the target in the search display (target present) and the other half did not (target absent). The percentage of color and orientation trials was manipulated across distractor contexts: The color-weighted distractor context consisted of $80 \%$ color trials and $20 \%$ orientation trials, whereas the orientationweighted distractor context consisted of $20 \%$ color trials and $80 \%$ orientation trials. (Color figure online)

\section{Results}

The accuracy and RTs data were entered into a repeatedmeasures ANOVA, with target presence (present, absent), trial type (color, orientation), and distractor context (color weighted, orientation weighted) as within-subjects factors. The critical interaction of interest was between trial type (color vs. orientation) and distractor context (color weighted vs. orientation weighted). We predicted relatively better performance on trials in which the trial type matched the probabilistic distractor context. Consistent with that expectation, the trial type $\times$ distractor context interaction was significant in both accuracy, $F(1,22)=6.38, p=.02, \eta_{p}^{2}=.23$, and RT, $F(1,22)$ $=50.68, p<.001, \eta_{p}^{2}=.70$ (see Fig. 2a). These interactions confirm the primary hypothesis that distractor context changes 

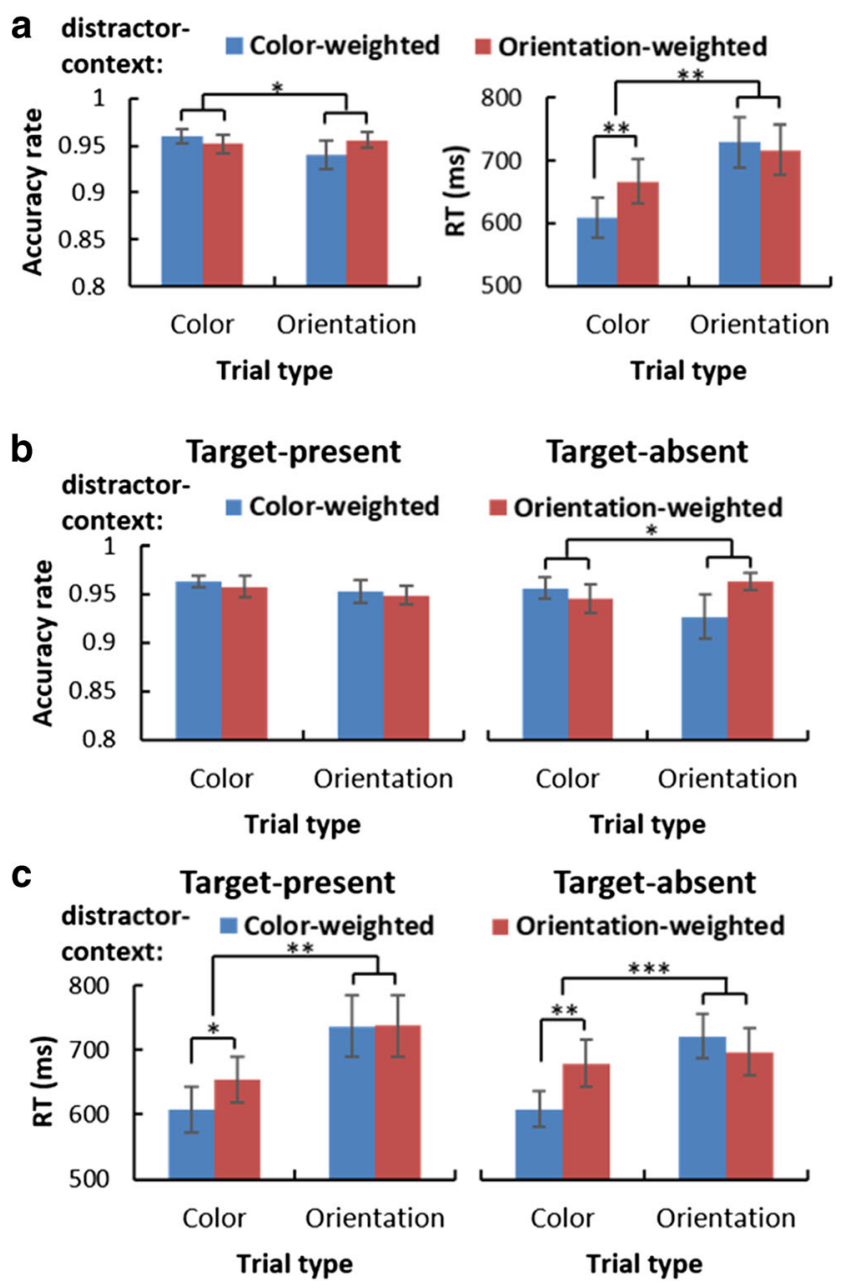

Fig. 2 Behavioral results in Experiment 1. $a$ Mean accuracy rate and RT for color and orientation trials in the color-weighted and orientationweighted distractor contexts. b Mean accuracy rate for color and orientation trials in the color-weighted and orientation-weighted distractor contexts shown separately for target-present and target-absent conditions. c Mean RT for color and orientation trials in the color-weighted and orientation-weighted distractor contexts, shown separately for targetpresent and target-absent conditions. Error bars represent the SEM in all figures. $* \mathrm{p}<.05, * * \mathrm{p}<.01, * * * \mathrm{p}<.001$

attentional priority for conjunction targets, but the relatively weaker effects on accuracy might be due to ceiling effects (see Fig. 2). Post hoc comparisons indicated that the interaction was due to the asymmetrical biases in the color-weighted and orientation-weighted distractor contexts: In the colorweighted distractor context, the mean accuracy rate was marginally higher for color trials (.96) than for orientation trials (.94) $(p=.07)$. In the orientation-weighted distractor context, the mean accuracy rates were not significantly different between color trials (.95) and orientation (.96) trials $(p=.61)$. RT was always shorter for color trials, but the advantage was bigger in the color-weighted distractor context $(121 \mathrm{~ms})$ than in the orientation-weighted distractor context $(51 \mathrm{~ms}), t(22)=$ $7.12, p<.001$.
The fact that the interaction pattern did not fully cross over suggests that color information was encoded more easily (automatically) and tended to pop out more than orientation overall. However, the fact that the "color advantage" was significantly diminished in the orientation-weighted distractor context suggests that probabilistic expectations about the feature dimension with greater diagnostic value significantly counteracted the default bias for color. This result indicates that the search performance was modulated by the current distractor context, and confirms our main hypothesis that probabilistic expectations bias attention toward the more diagnostic feature of the target, even when both target features are known in advance.

There were no other significant effects in accuracy $\left(F_{\mathrm{s}}<\right.$ $2.87, p s>.10$; see Fig. 2b). However, there were several other significant results in RT that were all related to the overall color advantage being modulated by other factors (see Fig. 2c). First, there was a significant main effect of trial type, $F(1,22)=47.61, p<.001, \eta_{p}^{2}=.68$, with a shorter mean RT for color trials $(637 \mathrm{~ms})$ than for orientation trials $(723 \mathrm{~ms})$, again indicating that color trials were relatively easier than orientation trials. There was also a significant two-way interaction between trial type and target presence, $F(1,22)=9.16$, $p=.006, \eta_{p}^{2}=.29$. Post hoc comparisons revealed that the RT advantage for color trials over orientation trials was bigger in target-present trials $(106 \mathrm{~ms})$ than in target-absent trials $(66$ $\mathrm{ms}), t(22)=3.03, p=.006$, again consistent with the color advantage. The three-way interaction between target presence, trial type, and distractor context was also significant, $F(1,22)$ $=4.95, p=.04, \eta_{p}^{2}=.18$, with greater trial type $\times$ distractor context interaction in target-absent than in target-present trials (see Fig. 2c). Other main effects and interactions in RT did not reach significance $(F \mathrm{~s}<1.47, p \mathrm{~s}>.24)$.

The results of this first experiment confirmed our primary hypothesis that the distractor context would modulate attentional weighting to target information in each of the two dimensions. The results also confirmed our suspicions that color and orientation would not be equally weighted. Interestingly, the interaction of interest was stronger in the target-absent trials. This difference was unexpected, given the search display of target-absent trials consisted of homogenous distractors for both color and orientation trials. However, the results overall suggest that participants biased attentional allocation toward the target feature from the more diagnostic dimension. If participants searched for a singleton from any feature dimension without using the distractor-contextspecific features presented in the cue, then there should be no difference in search performance between orientationtarget-absent and color-target-absent trials. The significant trial type $\times$ distractor context interactions observed therefore provide evidence that participants encoded the cue and modulated attentional gain for those specific features on each trial, 
rather than just relying on the singleton search mode despite an overall advantage for color.

\section{Experiment 2a}

The results in Experiment 1 provided evidence that a conjunction target's feature values are weighted by expectations about which feature dimension best distinguishes targets from distractors, even when the exact target features are known in advance. However, because the distractors were homogeneous in Experiment 1, it was still possible to perform the search task by finding the odd pop-out feature within one dimension without using the specific features in the cue (Liesefeld \& Müller, 2019). Thus, it is possible that the results observed in Experiment 1 were due to upweighting of saliency signals from the more diagnostic dimension, or even all feature signals from the diagnostic dimension, rather than selective upweighting of the cued feature in the more diagnostic dimension. In order to tease apart these possibilities, Experiment 2a investigated whether the dimensional bias could be applied to specific target features, by making distractors heterogeneous to prevent target pop-out. Since distractor items were now all heterogeneous, participants had to use the trial-specific cued target features in order to find the target (see Fig. 3). The question was whether participants will continue to preferentially use the feature value from the more informative dimension to bias attention during visual search.

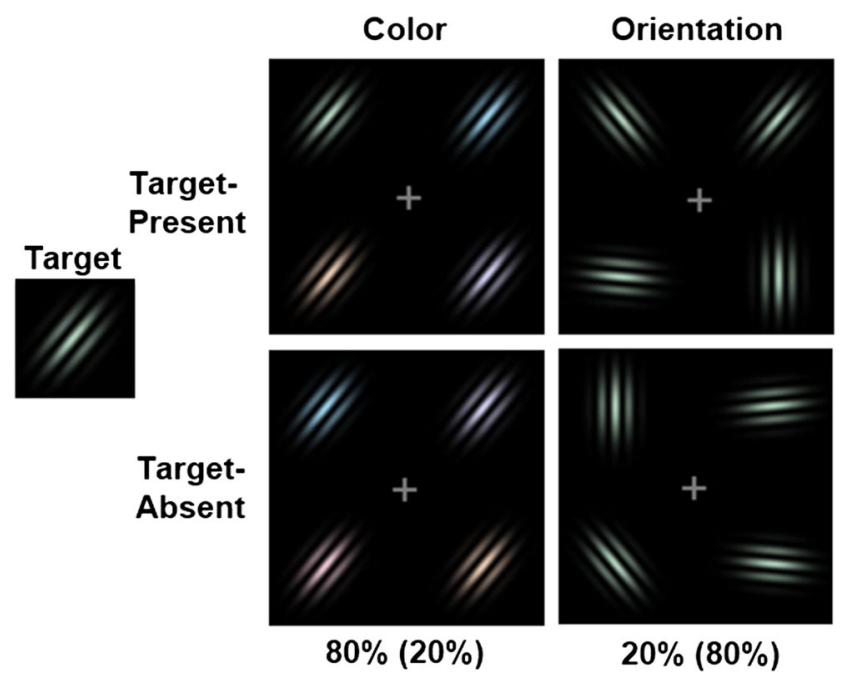

Fig. 3 Example search displays for different trial types in Experiment 2. The distractor items were all heterogeneous, inducing conjunction search. Trial types and the probability manipulations across the color-weighted and orientation-weighted distractor contexts were the same as in Experiment 1 . The probability manipulations were implicitly learned in Experiment 2a, and were explicitly told in Experiment $2 \mathrm{~b}$. (Color figure online)

\section{Method}

Participants Twenty-three participants engaged in Experiment 2a (10 males, mean age: 21.32 years, age range: $18-24$ years). The number of participants in each experiment was determined a priori using power analysis $\left(\mathrm{G}^{*}\right.$ Power, Version 3.1.9.2; http://www.gpower.hhu.de/), with .80 power to find a medium-size effect in a repeated-measures within-subjects factor ANOVA test. All had normal or corrected-to-normal vision and gave informed consent. All of the experimental procedures were approved by the Institutional Review Board of the University of California, Davis.

Design and procedures Experiment 2a was conducted to investigate whether expectations about the distractor context will lead to preferential weighting of features from targetdiagnostic dimensions, even when the target does not pop out. The stimuli and procedure in Experiment 2a were identical to those of Experiment 1, except that the distractor items were all heterogeneous (see Fig. 3): On color trials, the orientation of all stimuli were identical, but the color of each distractor item was unique and randomly selected from orange $\left(18.5 \mathrm{~cd} / \mathrm{m}^{2}, x=.42, y=.40\right)$, green $\left(18.8 \mathrm{~cd} / \mathrm{m}^{2}, x=.30, y=\right.$ $.44)$, blue $\left(18.9 \mathrm{~cd} / \mathrm{m}^{2}, x=.23, y=.28\right)$, purple $\left(18.2 \mathrm{~cd} / \mathrm{m}^{2}, x\right.$ $=.27, y=.25)$, and pink $\left(18.4 \mathrm{~cd} / \mathrm{m}^{2}, x=.37, y=.30\right.$; excluding the current target color) without replacement. The two additional distractor colors (purple and pink, 30 degrees apart from each other) were used in Experiments 2 and 3 to make all distractor items in a search display heterogeneous. In orientation trials, the color of all stimuli was identical, but the orientation of each distractor item was randomly chosen from $50^{\circ}$, $90^{\circ}, 130^{\circ}, 5^{\circ}$, and $175^{\circ}$ (excluding the current target orientation) without replacement. The two additional distractor orientations $\left(5^{\circ}\right.$ and $\left.175^{\circ}\right)$ were used in Experiments 2 and 3 to make all distractor items in a search display heterogeneous.

\section{Results}

The accuracy and RT data were entered into repeatedmeasures ANOVA, with target presence (present, absent), trial type (color, orientation), and distractor context (color weighted, orientation weighted) as within-subjects factors. First, there was a significant main effect of target presence in the RT data, $F(1,22)=41.50, p<.001, \eta_{p}^{2}=.65$, with faster RTs for target-present trials $(844 \mathrm{~ms})$ than for target-absent trials $(988 \mathrm{~ms})$. This confirms that this experimental task required serial search rather than pop-out search (cf. no Experiment 1 main effect of target presence in RT and accuracy).

As with Experiment 1, the interaction of primary interest between trial type and distractor context was significant in both accuracy, $F(1,22)=6.24, p=.02, \eta_{p}^{2}=.22$, and RT, $\mathrm{F}(1$, 22) $=21.83, p<.001, \eta_{p}^{2}=.50$, indicating that the search 

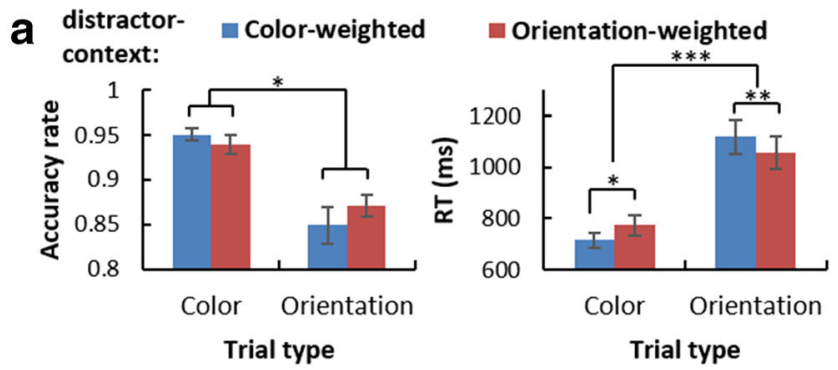

b

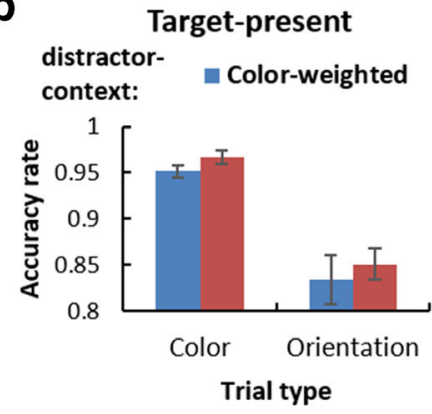

C distractorcontext:

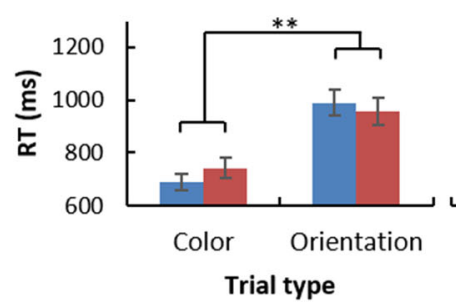

Target-present Color-weighted ted

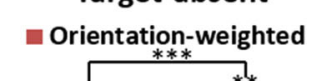

Target-absent

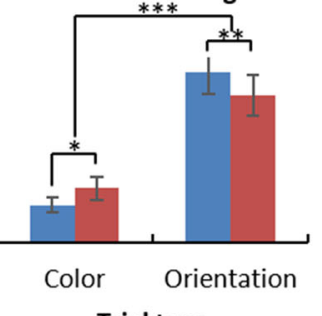

Trial type

Fig. 4 Behavioral results in Experiment 2a. a Mean accuracy rate and RT for color and orientation trials in the color-weighted and orientationweighted distractor contexts. b Mean accuracy rate for color and orientation trials in the color-weighted and orientation-weighted distractor contexts, shown separately for target-present and target-absent conditions. c Mean RT for color and orientation trials in the colorweighted and orientation-weighted distractor contexts, shown separately for target-present and target-absent conditions. $* p<.05$, ** $p<.01$, ***p $<.001$

performance was modulated by the distractor context (see Fig. 4a). Post hoc comparisons of both interactions indicated that the difference in performance between color and orientation trials (color advantage) was greater in the color-weighted distractor context $(.10 ; 404 \mathrm{~ms})$ than in orientation-weighted distractor context $(.07 ; 284 \mathrm{~ms}), t(22)=2.50, p=.02 ; t(22)=$ $4.67, p<.001$, respectively. These results replicate those of Experiment 1 in showing that while there is an overall bias toward color information, the distractor context modulated this effect according to whether color or orientation was the more diagnostic feature dimension.

Consistent with the overall bias for color found in the interaction as well as in the results of Experiment 1, there were significant main effects of trial type in the accuracy and RT data-accuracy: $F(1,22)=36.87, p<.001, \eta_{p}^{2}$

$=.63$; RT: $F(1,22)=80.10, p<.001, \eta_{p}^{2}=.79$ (see Fig. $4 \mathrm{~b}-$ c). Performance was better on color trials $(.95 ; 744 \mathrm{~ms})$ than in orientation trials $(.86 ; 1,088 \mathrm{~ms})$. There were also significant interactions between trial type and target presence in accuracy and RT-accuracy: $F(1,22)=5.55, p=.03, \eta_{p}^{2}=$ .20 ; RT: $F(1,22)=30.02, p<.001, \eta_{p}^{2}=.58$. There was a bigger difference in mean performance between the color and orientation trial types in target-present trials $(.12 ; 429 \mathrm{~ms})$ than in target-absent trials $(.05 ; 259 \mathrm{~ms})$-accuracy: $t(22)=$ $2.36, p=.03$; RT: $t(22)=5.48, p<.001$. Replicating Experiment 1, this suggests that the general color benefit was stronger when a target was actually present in the search display. Together, these results confirm that color trials were relatively easier than orientation trials in the current paradigm, even when the target did not pop out in one dimension. Other main effects or interactions did not reach significance $(F \mathrm{~s}<3.98, p \mathrm{~s}>.06)$.

\section{Experiment 2b}

One possible reason for the continued overall bias toward color was that we did not tell participants explicitly about the different distractor contexts. The stimuli and procedure in Experiment $2 \mathrm{~b}$ were identical to those of Experiment 2a, except that the participants were explicitly told about the trial type probability manipulation across distractor contexts.

\section{Method}

Participants Twenty-three participants engaged in Experiment 2b (seven males, mean age: 22.73 years, age range: $18-28$ years). The number of participants in each experiment was determined a priori using power analysis ( $\mathrm{G}^{*}$ Power, Version 3.1.9.2; http://www.gpower. hhu.de/), with .80 power to find a medium-size effect in a repeated-measures within-subjects factor ANOVA test. All had normal or corrected-to-normal vision and gave informed consent. All of the experimental procedures were approved by the Institutional Review Board of the University of California, Davis.

Design and procedures The stimuli and procedure in Experiment $2 \mathrm{~b}$ were identical to those of Experiment 2a, except that the participants were explicitly told about the trial type probability manipulations across distractor contexts: Before starting each distractor context condition, example search displays of the two trial types (color, orientation) were shown on the screen, and it was clearly stated which trial type will occur more frequently $(80 \%)$ for the next eight blocks. 


\section{Results}

Following the approach from Experiments 1 and 2a, the accuracy and RT data were entered into a repeated-measures ANOVA, with target presence (present, absent), trial type (color, orientation), and distractor context (color weighted, orientation weighted) as within-subjects factors. As in Experiment 2a, mean RT was significantly shorter on target-present (824 $\mathrm{ms}$ ) than on target-absent trials (963 ms), $F(1,22)=81.56, p<$ $.001, \eta_{p}^{2}=.79$. This again confirms that the design required serial search through the displays (cf. Experiment 1).

With respect to the main interaction of interest between trial type and distractor context (see Fig. 5a), it was surprising that when subjects were explicitly told about the probabilities of
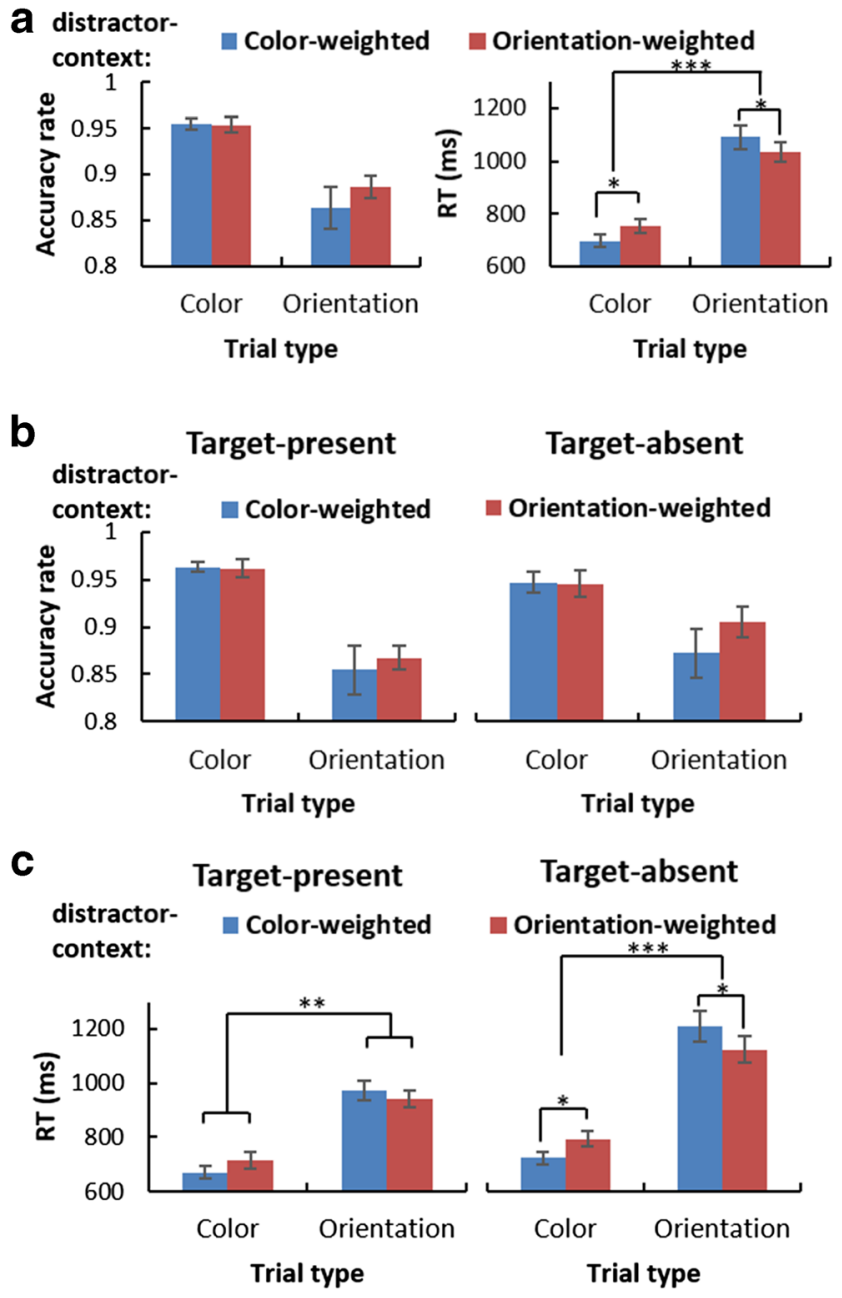

Fig. 5 Behavioral results in Experiment 2b. b Mean accuracy rate and RT for color and orientation trials in the color-weighted and orientationweighted distractor contexts. b Mean accuracy rate for color and orientation trials in the color-weighted and orientation-weighted distractor contexts, shown separately for target-present and target-absent conditions. c Mean RT for color and orientation trials in the colorweighted and orientation-weighted distractor contexts, shown separately for target-present and target-absent conditions. $* \mathrm{p}<.05, * * \mathrm{p}<.01$, ***p $<.001$ trial types within each distractor context, the interaction was only significant in RT-accuracy: $F(1,22)=1.67, p=.21$; RT: $F(1,22)=25.14, p<.001, \eta_{p}^{2}=.53$. The difference in mean RT between color and orientation trials (color advantage) was again greater in the color-weighted distractor context $(395 \mathrm{~ms})$ than in the orientation-weighted distractor context $(280 \mathrm{~ms}), t(22)=5.01, p<.001$. The RT effect replicates Experiments 1 and 2a. The lack of an effect in accuracy may have been spurious given the overall similarity in the pattern of data, due to ceiling effects, or due to a stronger explicit bias toward color (i.e., accuracy on color trials in both distractor context conditions was uniformly high).

Replicating both previous experiments, in addition to the interaction of interest, there was a main effect of trial type in both accuracy, $F(1,22)=25.63, p<.001, \eta_{p}^{2}=.54$, and RT, $F(1,22)=205.56, p<.001, \eta_{p}^{2}=.90$ (see Fig. 5b-c). Accuracy was higher (.95) and RT shorter (725 ms) on color trials than on orientation trials (.88 and $1,062 \mathrm{~ms}$, respectively). This reinforces the notion that participants tended to rely on color more than on orientation overall. Similarly, replicating Experiment 2a, there were also significant interactions between trial type and target presence in accuracy, $F(1,22)=$ $4.65, p=.04, \eta_{p}^{2}=.18$, and RT, $F(1,22)=27.22, p<.001, \eta_{p}^{2}=$ .55 . The RT effect was modulated by a three-way interaction between trial type, target presence, and distractor context, $F(1$, 22) $=4.85, p=.04, \eta_{p}^{2}=.18$, that indicated stronger trial type $\times$ distractor context interaction on target-absent trials than on target-present trials. Other main effects or interactions did not reach significance $\left(F_{\mathrm{s}}<1.00, p \mathrm{~s}>.33\right)$.

The pattern of results obtained in Experiment $2 b$ was very similar to that of Experiment $2 \mathrm{a}$. This was verified statistically by entering data from Experiments $2 \mathrm{a}$ and $2 b$ in a mixed ANOVA, with experiment (Experiment 2a, Experiment 2b) as a between-subjects factor, and target presence (present, absent), trial type (color, orientation), and distractor context (color weighted, orientation weighted) as within-subjects factors. The results revealed no significant interaction involving experiment, either in accuracy or RT data $\left(F_{\mathrm{S}}<1\right)$. The same data was also examined using Bayesian statistics (JASP, Version 0.9; https://jasp-stats.org/2018/06/20/ introducing-jasp-0-9/), comparing the fit of the data under the null hypothesis and the alternative hypothesis. The results obtained from Bayesian statistics were consistent with those from frequentist statistics: For accuracy, the inclusion Bayes factor $\left(\mathrm{BF}_{\text {inclusion}}\right)$ across matched models for the trial type $\times$ distractor context $\times$ Experiment interaction was 0.22, suggesting that the evidence for the three-way interaction was 0.22 , or rather, it was $4.55(1 / 0.22)$ times more likely to occur under a model without including the three-way interaction. For $\mathrm{RT}$, the $\mathrm{BF}_{\text {inclusion }}$ across 
matched models for the trial type $\times$ distractor context $\times$ Experiment interaction was 0.21 , suggesting that it was $4.76(1 / 0.21)$ times more likely to occur under a model without including the three-way interaction. These results suggest that it did not matter whether the information about the changing distractor contexts was given explicitly or learned implicitly.

\section{Experiment 3}

The results of Experiments 1 and 2 provided clear evidence that attentional biases for features of a conjunction target are weighted by the likelihood of that feature dimension being diagnostic of the target within the current distractor context. We next examined the effect of increasing the set size on relative weighting for each feature of a conjunction target (see Fig. 6). We hypothesized that if relative weighting for each feature dimension is determined by top-down expectations about the diagnosticity of feature dimensions, then simply increasing the set size would not change the relative weightings per se. In order to increase the set size without changing the overall contrast between the target and distractor items, the number of possible distractor features were held constant at five, instead of adding new possible distractor features. That is, the effective number of distractors (in terms of alternative within-dimension features) was held constant across set sizes, and therefore we expected the overall contrast between the target and distractor items was the same across set sizes.

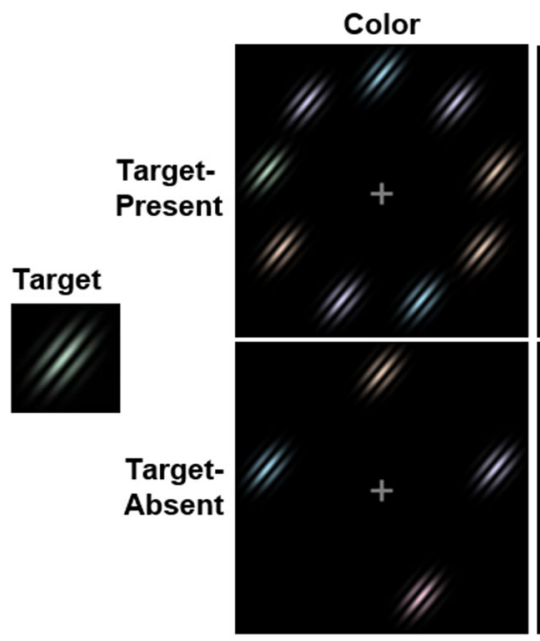

$80 \%(20 \%)$

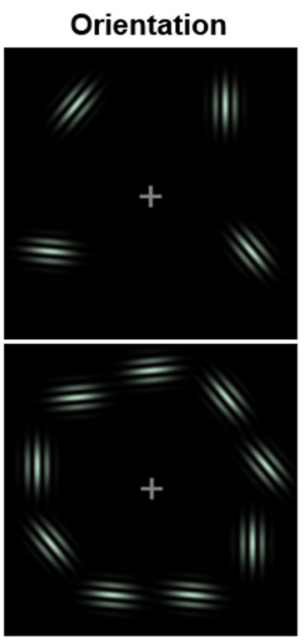

$20 \%(80 \%)$
Fig. 6 Example search displays for different trial types in Experiment 3. The set size of items in the search display was manipulated to be four in half of the trials, and nine in other half of the trials. Trial types and the probability manipulations across the color-weighted and orientationweighted distractor contexts were identical as in Experiment 2. Each participant performed either the color-weighted distractor-context blocks or orientation-weighted distractor-context blocks, counterbalanced across participants. (Color figure online)

\section{Method}

Participants Thirty-six participants (eight males, mean age: 21.39 years, age range: 18-32) engaged in a 1-hour session for course credit. The number of subjects was determined a priori using power analysis $\left(\mathrm{G}^{*}\right.$ Power, Version 3.1.9.2; http:// www.gpower.hhu.de/), with .80 power to find a medium-size effect in a repeated-measures between-factors ANOVA test. All had normal or corrected-to-normal vision and gave informed consent. All of the experimental procedures were approved by the Institutional Review Board of the University of California, Davis.

\section{Design and procedures}

The stimuli and procedure in Experiment 3 were identical to those of Experiment 2, except that the number of items in the search display could be four (as in previous experiments) in half of the trials or nine in the other half (see Fig. 6). As in Experiments $2 \mathrm{a}-\mathrm{b}$, the feature of each distractor item was randomly selected from five colors (orange, green, blue, purple, and pink) or five orientations $\left(50^{\circ}, 90^{\circ}, 130^{\circ}, 5^{\circ}\right.$, and $175^{\circ}$ ) without replacement (excluding the current target feature). The location of each item in the search display was randomly chosen among nine equidistant positions that were on the rim of an imaginary circle with a radius of $3.8^{\circ}$. The manipulation of dimension-weighting distractor context (previously within subject) was now manipulated between subjects due to the increase in trial numbers with two set sizes. Each participant performed either the color-weighted distractor context blocks or orientation-weighted distractor context blocks (40 trials/block, 14 blocks), counterbalanced across participants.

\section{Results}

The accuracy and RT data were entered into repeatedmeasures ANOVA, with set size $(4,9)$, target presence (present, absent), and trial type (color, orientation) as withinsubjects factors, and distractor context (color weighted, orientation weighted) as a between-subjects factor.

Consistent with the previous experiments, the interaction between trial type and distractor context was significant in accuracy, $F(1,34)=5.08, p=.03, \eta_{p}^{2}=.13$, and RT, $F(1$, $34)=4.78, p=.04, \eta_{p}^{2}=.12$ (see Fig. 7a). Post hoc comparisons revealed that the difference in mean accuracy and RT between color and orientation trials (color advantage) was bigger in the color-weighted distractor context $(.06 ; 840 \mathrm{~ms})$ than in the orientation-weighted distractor context $(.04 ; 665$ $\mathrm{ms}), t(34)=2.26, p=.03 ; t(34)=2.19, p=.04$, indicating that despite the prepotent effect of color on target selection, information from the two dimensions were differentially weighted 
a

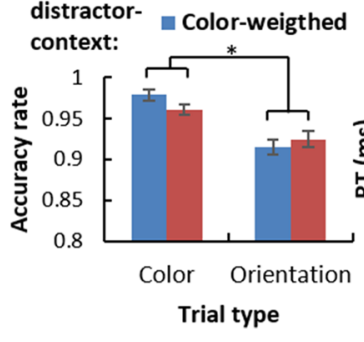

- Orientation-weighted

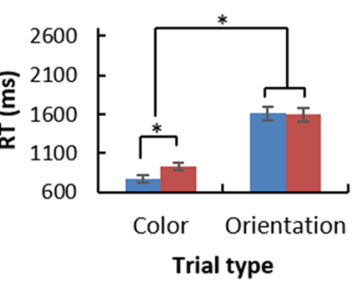

b
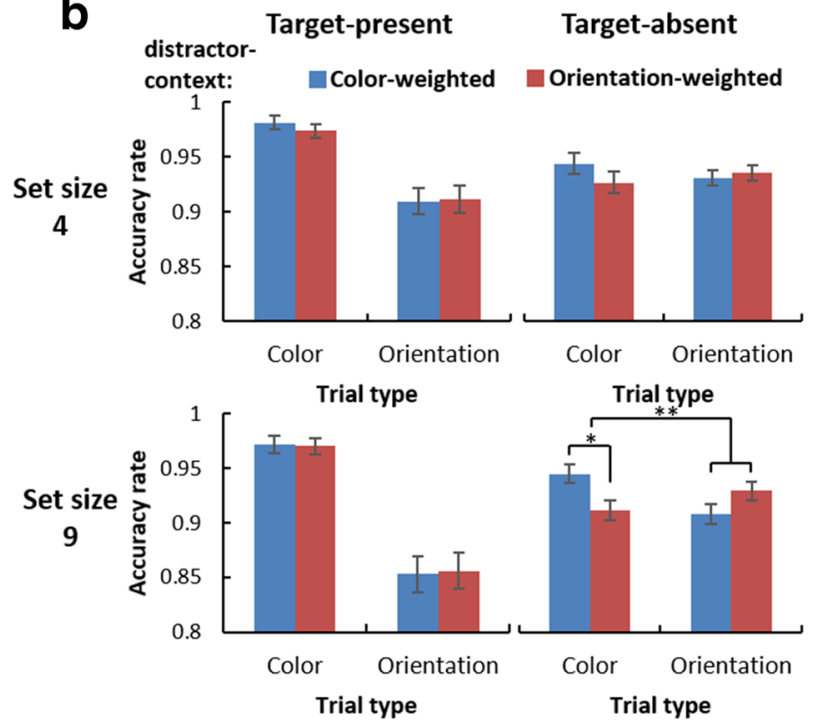

Fig. 7 Behavioral results in Experiment 3. a Mean accuracy rate and RT for color and orientation trials in the color-weighted and orientationweighted distractor contexts. b Mean accuracy rate for color and orientation trials in the color-weighted and orientation-weighted distractor contexts, shown separately for the target presence (present,

according to distractor context. Set size did not interact with these two factors; the three-way interaction between trial type, distractor context, and set size was not significant in accuracy or RT-accuracy: $F=1.26, p=.27$; RT: $F=2.19, p=.15-$ indicating that the effect of dimensional weighting according to distractor context was not affected by set size. Bayesian statistics (JASP, Version 0.9; https://jasp-stats.org/2018/06/ 20/introducing-jasp-0-9/) supported interpretation of the null interaction: For accuracy, the $\mathrm{BF}_{\text {inclusion }}$ across matched models for the trial type $\times$ distractor context $\times$ set size interaction was 0.40 , suggesting that the data was $2.50(1 / 0$. 40) times more likely to occur under a model without including the three-way interaction. For $\mathrm{RT}$, the $\mathrm{BF}_{\text {inclusion }}$ across matched models for the trial type $\times$ distractor context $\times$ set size interaction was 0.26 , suggesting that the data was 3 . $85(1 / 0.26)$ times more likely to occur under a model without the three-way interaction.

In addition to the main results of interest, we again found the overall color advantage from previous experiments. The main effect of trial type was significant in both accuracy and RT—accuracy: $F(1,34)=66.73, p<.001, \eta_{p}^{2}=.66$; RT: $F(1$, $34)=355.37, p<.001, \eta_{p}^{2}=.91$ - with better performance for

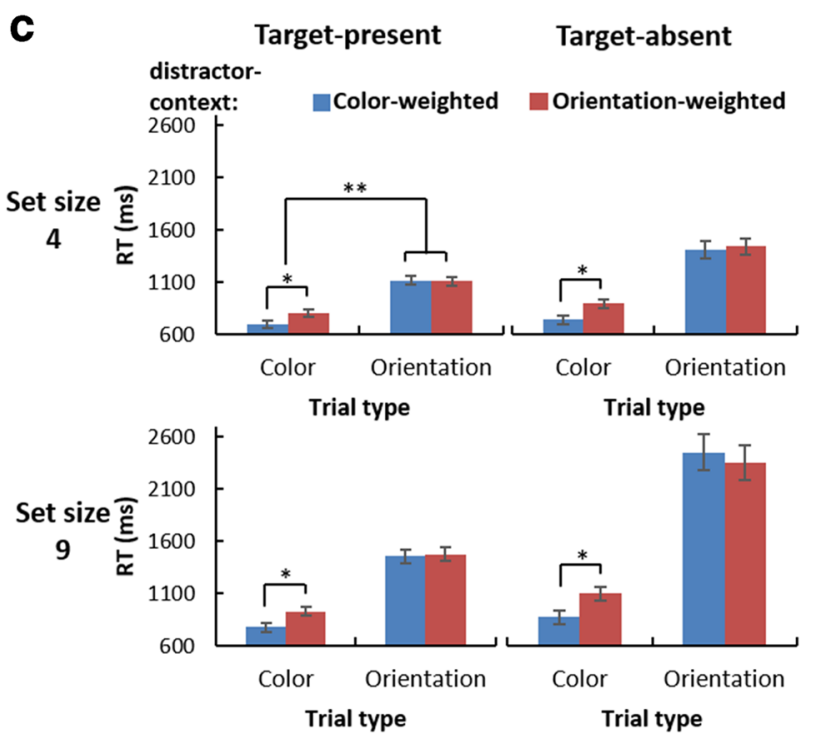

absent) and set size $(4,9)$ conditions. c Mean RT for color and orientation trials in the color-weighted and orientation-weighted distractor contexts, shown separately for the target presence (present, absent) and set size $(4,9)$ conditions. $* p<.05, * * p<.01$

color trials $(.97 ; 851 \mathrm{~ms})$ than for orientation trials $(.92 ; 1603$ $\mathrm{ms}$ ) (see Fig. 7b-c). Also similar to Experiment 2, the main effect of target presence was significant-accuracy: $F(1,34)=$ $50.40, p<.001, \eta_{p}^{2}=.60$; RT: $F(1,34)=109.39, p<.001, \eta_{p}^{2}=$ .76 - with better performance for target-present trials (.96; $1045 \mathrm{~ms})$ than for target-absent trials $(.93 ; 1409 \mathrm{~ms})$. Finally, the main effect of set size was significant-accuracy: $F(1,34)$ $=27.66, p<.001, \eta_{p}^{2}=.45$; RT: $F(1,34)=214.59, p<.001, \eta_{p}^{2}$ $=.86$-with better performance for Set Size $4(.96 ; 1,028 \mathrm{~ms})$ than for Set Size 9 (.93; $1426 \mathrm{~ms})$, suggesting that the manipulation of set size was effective overall in increasing search difficulty.

Finally, consistent with the notion that color dominates target selection, the two-way interaction between trial type and set size was significant in both accuracy and RT-accuracy: $F(1,34)=13.32, p<.001, \eta_{p}^{2}=.28$; RT: $F(1,34)=$ 207.94, $p<.001, \eta_{p}^{2}=.86$ - with a bigger difference in performance between Set Sizes 4 and 9 on orientation trials (.04; $664 \mathrm{~ms})$ than on color trials $(.01 ; 133 \mathrm{~ms})$-accuracy: $t(35)=$ $3.64, p<.001$; RT: $t(35)=14.18, p<.001$. Similarly, there were also significant interactions between trial type and target 
presence-accuracy: $F(1,34)=60.66, p<.001, \eta_{p}^{2}=.64$; RT: $F(1,34)=91.59, p<.001, \eta_{p}^{2}=.73$ - between target presence and set size - accuracy: $F(1,34)=6.21, p=.02, \eta_{p}^{2}=.15$; RT: $F(1,34)=82.52, p<.001, \eta_{p}^{2}=.71$-and between trial type, target presence, and set size-accuracy: $F(1,34)=8.84, p=$ $.005, \eta_{p}^{2}=.21 ; \mathrm{RT}: F(1,34)=82.91, p<.001, \eta_{p}^{2}=.71$.

\section{Discussion}

The core function of attention is to efficiently select and enhance a subset of currently relevant information while minimizing the effect of distracting information. Theories of attention hypothesize that attentional priority is established by summing the signals from feature-selective maps (Cave \& Wolfe, 1990; Treisman \& Gelade, 1980; Wolfe, 1994), and that attention enhances the representation of critical information by increasing the activity of neurons tuned to relevant features (Bichot, Rossi, \& Desimone, 2005; Boynton, 2005; Desimone \& Duncan, 1995; Hamker, 2004; Martinez-Trujillo \& Treue, 2004; Treue \& Martinez Trujillo, 1999). In addition to the feature-based enhancement models, the dimensionweighting account further hypothesizes that information from each feature dimension is weighted based on expectations of how informative each feature dimension will be (Müller et al., 1995; Töllner et al., 2012; Töllner et al., 2010). However, these theories posit two different optimal contexts - namely, when the exact target features are known in advance versus when the target pops out within a single feature dimension. Few studies have examined the intersection between featurebased selection and dimensional weighting. The current set of experiments addressed the question of whether feature-based enhancement and dimensional weighting can co-occur when the exact target features are known, but one dimension is more likely to be diagnostic of the target during visual search.

The results of the current study indicated that dimensional weights were flexibly adjusted according to distractor context, even when the exact cue of a color-orientation conjunction target was presented at every trial and the target did not pop out along a single feature dimension. The interaction between trial type and distractor context was replicated across four experiments, providing strong evidence that dimensional weighting and feature-based enhancement co-occur. The observed modulation of search performance could have originated from two different mechanisms: One would occur early at the presentation of a cue, by selectively encoding only the more diagnostic feature of the cue. Another mechanism would occur at the presentation of a search array, by increasing sensory enhancement for the feature value that belongs to the more diagnostic feature dimension. An extreme version of selectively encoding only the feature that is expected to be more informative is unlikely. First, the accuracy rate was overall very high (more than $90 \%$ ) on both valid and invalid trials, suggesting that both features of the cue were correctly encoded on most trials. Second, the effect size of interaction between trial type and distractor context was bigger in the RT data than in the accuracy data across multiple experiments. Considering that only correct trials were included for analysis in the RT data, the interaction observed in the RT data cannot be explained by trial-to-trial variability in "all-or-none" encoding of cue features. Thus, it is highly unlikely that selective encoding of only one cue feature was solely responsible for the observed modulation of search performance, but it is not possible to rule out a version in which encoding of the two features was biased toward the more diagnostic feature (Witkowski \& Geng, 2019). These results overall fit well with the hypothesis that feature-based enhancement is weighted by expectations about the diagnosticity of feature dimensions.

Regarding how feature-based enhancement is weighted by expectations about the diagnosticity of feature dimensions, there are several possibilities: Selective enhancement could have occurred for the target feature within the more diagnostic dimension on the current trial, all possible target features within the diagnostic dimension, or all features within the diagnostic dimension (e.g., dimensional weighting). However, in the current experiments, target and distractor features were randomly chosen from the same pool of possible features on every trial. Thus, uniformly enhancing all possible target features or all features in the more diagnostic dimension would not provide a selective advantage for discriminating the target from distractors. Therefore, the observed modulation in search performance according to distractor context is likely due to selective enhancement of the current target feature within the more diagnostic dimension (e.g., either by targeting the single feature value, or by summing separate weights for the dimension and feature).

Another consistent finding throughout experiments is that the overall attentional biases were not equivalent for different feature dimensions (color, orientation) during conjunction target search. The main effect of trial type was replicated across all experiments, with better search performance for color trials than for orientation trials. The color and orientation feature values used in the current study were selected to have equal distance between each value in their respective feature spaces (i.e., $0^{\circ}-360^{\circ}$ on a color wheel, $0^{\circ}-180^{\circ}$ in orientation), but it is likely that the perceptual similarity between feature values in the two dimensions were not equated (Duncan \& Humphreys, 1989). In addition, other studies have also found that color generally enjoys greater attentional priority than other feature dimensions, such as shape (Biderman et al., 2017; Geng et al., 2017; Lee et al., 2018; Theeuwes, 1991, 1992; White et al., 2014), and may have more complex nonlinear properties than other dimensions (Found \& Müller, 1996; Liesefeld, Liesefeld, Pollmann, \& Müller, 2018; 
Müller et al., 2003). Regardless of the reason that color tends to have precedence in attentional priority over other feature dimensions, the critical result in the current study was that the size of the overall color advantage over orientation was modulated by probabilistic distractor context. This indicates that expectations about the informativeness of a feature dimension modulate preexisting biases in attentional priority.

Taken together, the current study provides evidence that expectations about the more informative dimension can be used to weight feature-based attentional priority to optimize search performance. Moreover, this form of dimensional weighting occurred irrespective of whether the information about the distractor context was learned implicitly or explicitly. This suggests that the probabilistic context of distractors is rapidly acquired and used to modulate the attentional priority of feature-selective maps in order to optimize search performance.

Acknowledgements This work was funded by NSF BCS $1230377-0$ to J.J.G. The authors declare no competing financial interests. The data and materials for all experiments are available upon request, and none of the experiments was preregistered.

\section{References}

Bae, G. Y., Olkkonen, M., Allred, S. R., \& Flombaum, J. I. (2015). Why some colors appear more memorable than others: A model combining categories and particulars in color working memory. Journal of Experimental Psychology: General, 144(4), 744-763. doi:https:// doi.org/10.1037/xge0000076

Bichot, N. P., Rossi, A. F., \& Desimone, R. (2005). Parallel and serial neural mechanisms for visual search in macaque area V4. Science, 308(5721), 529-534. doi:https://doi.org/10.1126/science.1109676

Biderman, D., Biderman, N., Zivony, A., \& Lamy, D. (2017). Contingent capture is weakened in search for multiple features from different dimensions. Journal of Experimental Psychology: Human Perception and Performance, 43(12), 1974-1992. doi:https://doi. org/10.1037/xhp0000422

Boynton, G. M. (2005). Attention and visual perception. Current Opinion in Neurobiology, 15(4), 465-469. doi:https://doi.org/10.1016/j. conb.2005.06.009

Cave, K. R., \& Wolfe, J. M. (1990). Modeling the role of parallel processing in visual search. Cognitive Psychology, 22, 225-271.

Desimone, R., \& Duncan, J. (1995). Neural mechanisms of selective visual attention. Annual Review of Neuroscience, 18, 193-222.

Duncan, J., \& Humphreys, G. W. (1989). Visual search and stimulus similarity. Psychological Review, 96(3), 433-458.

Found, A., \& Müller, H. J. (1996). Searching for unknown feature targets on more than one dimension: Investigating a "dimensionweighting” account. Perception \& Psychophyics, 58(1), 88-101. doi:https://doi.org/10.3758/bf03205479

Geng, J. J., DiQuattro, N. E., \& Helm, J. (2017). distractor probability changes the shape of the attentional template. Journal of Experimental Psychology: Human Perception and Performance, 43(12), 1993-2007. doi:https://doi.org/10.1037/xhp0000430

Hamker, F. H. (2004). A dynamic model of how feature cues guide spatial attention. Vision Research, 44(5), 501-521. doi:https://doi.org/10. 1016/j.visres.2003.09.033
Krummenacher, J., \& Müller, H. (2012). Dynamic weighting of feature dimensions in visual search: Behavioral and psychophysiological evidence. Frontiers in Psychology, 3(221). doi:https://doi.org/10. 3389/fpsyg.2012.00221

Lee, J., Leonard, C. J., Luck, S. J., \& Geng, J. J. (2018). Dynamics of feature-based attentional selection during color-shape conjunction search. Journal of Cognitive Neuroscience, 30(12), 1773-1787. doi: https://doi.org/10.1162/jocn_a_01318

Liesefeld, H. R., Liesefeld, A. M., Pollmann, S., \& Müller, H. J. (2018). Biasing allocations of attention via selective weighting of saliency signals: Behavioral and neuroimaging evidence for the dimensionweighting account. Current Topics in Behavioral Neurosciences. doi:https://doi.org/10.1007/7854_2018_75

Liesefeld, H. R., \& Müller, H. J. (2019). Distractor handling via dimension weighting. Current Opinion in Psychology, 29, 160-67. doi: https://doi.org/10.1016/j.copsyc.2019.03.003

Martinez-Trujillo, J. C., \& Treue, S. (2004). Feature-based attention increases the selectivity of population responses in primate visual cortex. Current Biology, 14(9), 744-751. doi:https://doi.org/10. 1016/j.cub.2004.04.028S0960982204002684

Maunsell, J. H., \& Treue, S. (2006). Feature-based attention in visual cortex. Trends in Neurosciences, 29(6), 317-322. doi:10.1016/ j.tins.2006.04.001

Müller, H. J., Heller, D., \& Ziegler, J. (1995). Visual search for singleton feature targets within and across feature dimensions. Perception \& Psychophysics, 57(1), 1-17. doi:https://doi.org/10.3758/ BF03211845

Müller, H. J., Reimann, B., \& Krummenacher, J. (2003). Visual search for singleton feature targets across dimensions: Stimulus- and expectancy-driven effects in dimensional weighting. Journal of Experimental Psychology: Human Perception and Performance, 29(5), 1021-1035. doi:https://doi.org/10.1037/0096-1523.29.5. 1021

Theeuwes, J. (1991). Cross-dimensional perceptual selectivity. Perception \& Psychophysics, 50(2), 184-193.

Theeuwes, J. (1992). Perceptual selectivity for color and form. Perception \& Psychophysics, 51(6), 599-606.

Töllner, T., Müller, H. J., \& Zehetleitner, M. (2012). Top-down dimensional weight set determines the capture of visual attention: Evidence from the PCN component. Cerebral Cortex, 22(7), 1554-1563. doi:https://doi.org/10.1093/cercor/bhr231

Töllner, T., Zehetleitner, M., Gramann, K., \& Müller, H. J. (2010). Topdown weighting of visual dimensions: behavioral and electrophysiological evidence. Vision Res, 50(14), 1372-1381. doi:https://doi. org/10.1016/j.visres.2009.11.009

Treisman, A., \& Gelade, G. (1980). A feature-integration theory of attention. Cognitive Psychology, 12(1), 97-136.

Treisman, A., \& Sato, S. (1990). Conjunction search revisited. Journal of Experimental Psychology: Human Perception and Performance, 16(3), 459-478.

Treue, S., \& Martinez Trujillo, J. C. (1999). Feature-based attention influences motion processing gain in macaque visual cortex. Nature, 399(6736), 575-579.

Weidner, R., \& Müller, H. J. (2009). Dimensional weighting of primary and secondary target-defining dimensions in visual search for singleton conjunction targets. Psychological Research, 73(2), 198-211. doi:https://doi.org/10.1007/s00426-008-0208-9

White, A. L., Lunau, R., \& Carrasco, M. (2014). The attentional effects of single cues and color singletons on visual sensitivity. Journal of Experimental Psychology: Human Perception and Performance, 40(2), 639-652. doi:https://doi.org/10.1037/a0033775

Witkowski, P. W., \& Geng, J. J. (2019). Learned feature variance is encoded in the target template and drives visual search. Visual Cognition. Advance online publication. doi:https://doi.org/10. 1080/13506285.2019.1645779 
Wolfe, J. M. (1994). Guided Search 2.0: A revised model of visual search. Psychonomic Bulletin \& Review, 1, 202-238.

Wolfe, J. M. (2007). Guided Search 4.0: Current progress with a model of visual search. In W. D. Gray (Ed.). Integrated models of cognitive systems (pp. 99-119). New York, NY: Oxford University Press.

Wolfe, J. M., Cave, K. R., \& Franzel, S. L. (1989). Guided search: an alternative to the feature integration model for visual search. Journal of Experimental Psychology: Human Perception and Performance, 15(3), 419-433.
Wolfe, J. M., \& Horowitz, T. S. (2004). What attributes guide the deployment of visual attention and how do they do it? Nature Reviews Neuroscience, 5, 495. doi:https://doi.org/10.1038/nrn1411

Publisher's note Springer Nature remains neutral with regard to jurisdictional claims in published maps and institutional affiliations. 